\title{
Eficacia de la WebQuest para el aprendizaje cooperativo
}

\author{
Sonia Lara, Charo Repáraz
}

Departamento de Educación, Universidad de Navarra

\section{España}

slara@unav.es

Sonia Lara. Departamento de Educación. Universidad de Navarra. 31080 Pamplona. Spain.

E-mail: slara@unav.es

(C) Education \& Psychology I+D+i and Editorial EOS (Spain) 


\section{Resumen}

El estudio que presentamos está enmarcado en un proyecto más amplio que lleva a cabo el Colegio Erain (San Sebastián) desde febrero-marzo de 2004. Se trata de un proyecto centrado en la utilización educativa del vídeo digital. El proyecto ha sido catalogado como Proyecto I+D+i por la Diputación de Guipúzcoa y el Gobierno Vasco, que colaboran en su financiación junto con el Fondo Europeo de Desarrollo Regional.

En concreto, presentamos la introducción de la WebQuest como una herramienta que permite el trabajo cooperativo y autónomo de los alumnos en la realización de vídeos científicos. Nuestra pregunta de investigación fue la siguiente: ¿La WebQuest fomenta el trabajo cooperativo y autónomo de los alumnos de secundaria (16 años) cuando realizan un vídeo científico? Para responder a esta pregunta trabajamos con un grupo de alumnos de $4^{\circ}$ de ESO que debían producir un vídeo científico sobre la geografía de Guipúzcoa, en la asignatura de Geografía. Para ello, siguiendo las indicaciones dadas en una WebQuest, realizaron un trabajo de investigación y después el vídeo (http://www.erain.es/vde/produccion3.asp). La recogida de datos se ha llevado a cabo a través de una encuesta específicamente preparada para recoger datos sobre este contexto de aprendiazje colaborativo. Las conclusiones de este estudio son similares a las recogidas en la investigación sobre el aprendizaje cooperativo en lo que se refiere a variables tanto cognitivas como afectivas. En este sentido se dibujan algunas líneas de investigación futuras para profundizar en el estudio de la eficacia de la WebQuest para fomentar el aprendizaje autónomo y cooperativo.

Palabras clave: WebQuest; Aprendizaje cooperativo; Vídeo Digital; Aprendizaje basado en la investigación; Educación Secundaria.

Recepción: 07-09-07 Aceptación provisional: 01-10-07 Aceptación definitiva: 08-10-07 


\section{Introducción ${ }^{1}$}

Actualmente nos encontramos ante el reto de fomentar en nuestros alumnos habilidades y competencias relacionadas entre otras con la capacidad de análisis, síntesis y evaluación de la información, capacidad para manejar grandes cantidades de información que fácilmente tienen los alumnos a su disposición en esta Sociedad de la Información, capacidad para usar las Tecnologías de la Información y la Comunicación (TIC), capacidad crítica y capacidad para trabajar cooperativamente. El uso de WebQuest se muestra como una alternativa para fomentar un aprendizaje activo y cooperativo usando información de internet (Dodge, 1995; Repáraz, Lara, Mir y Orobiogoikoetxea, 2006; Lara, 2003).

El estudio que presentamos está enmarcado en un proyecto más amplio que lleva a cabo el Colegio Erain (San Sebastián) desde febrero-marzo de 2004. Se trata de un proyecto centrado en la utilización educativa del vídeo digital. El proyecto ha sido catalogado como Proyecto I+D+i por la Diputación de Guipúzcoa y el Gobierno Vasco, que colaboran en su financiación junto con el Fondo Europeo de Desarrollo Regional. El Proyecto consiste en la creación de un Portal de Internet de vídeo digital educativo, utilizando para ello las más avanzadas tecnologías multimedia en la edición y publicación de vídeo en Internet en tiempo real (Repáraz, Lara, Mir y Orobiogoikoetxea, 2006).

Gracias a la confluencia de los intereses del director del Colegio Erain y de las autoras de este trabajo se inicia el estudio que presentamos en este artículo. Por un lado, el Director del Colegio estaba buscando una forma de mejorar las pautas que daban a los alumnos para realizar los vídeos de investigación, y por otro lado las autoras querían investigar la eficacia de la WebQuest para fomentar el aprendizaje cooperativo y el aprendizaje autónomo. Tras varias reuniones de trabajo en las que se expusieron los intereses de ambas partes se planteo la posibilidad de introducir la WebQuest en el Proyecto $\mathrm{I}+\mathrm{D}+\mathrm{i}$ del Colegio Erain (http://www.videodigitaleducativo.com/).

La finalidad de introducir la WebQuest fue emplear una herramienta que permitiera el trabajo autónomo y cooperativo de los alumnos en la realización de vídeos científicos. El problema que el Director del Colegio planteó fue que los estudiantes se centraban en el uso del

\footnotetext{
${ }^{1}$ Una version abreviada de este artículo fué presentada a un congreso: Lara, S. y Repáraz, Ch. Effectiveness of Cooperative Learning: WebQuest as a Tool to Produce Scientific Videos. Paper presented In International Conference on Multimedia
} 
vídeo y se olvidaban de una parte muy importante, el trabajo previo de investigación. Los estudiantes no se organizaban bien ni con los medios audiovisuales e informáticos ni con el tiempo establecido para realizar el trabajo. En este contexto propusimos el empleo de la WebQuest como un buen medio para ayudar a los estudiantes a trabajar autónoma y cooperativamente gracias a las guías (andamiaje) que podrían ofrecérseles. Nuestra pregunta de investigación fue la siguiente: ¿La WebQuest fomenta el trabajo cooperativo y autónomo de los alumnos de secundaria (16 años) cuando realizan un vídeo científico?

Las funciones desempeñadas por ambas partes fueron las siguientes. Las autoras de este artículo adaptaron las principales pautas, que hasta el momento se daban a los alumnos para realizar los vídeos, a la metodología de la WebQuest. Elaboraron unas encuestas para recoger las opiniones de los alumnos una vez finalizada la elaboración de los vídeos, así como los análisis y el informe de resultados que presentamos en este artículo. El Director del Colegio eligió el grupo de alumnos y el profesor que participarían en la investigación, el cual siguió las pautas que las autoras dimos en la aplicación de la WebQuest.

Con este estudio hemos realizado una investigación empírica preliminar de corte descriptivo para intentar dar una respuesta al problema planteado por el Colegio Erain. Por tanto, el principal propósito fue ayudarles a resolver una situación de enseñanza-aprendizaje en la que los alumnos debían trabajar de forma autorregulada y cooperativa. Esta investigación que responde a un tipo de investigación-acción, tiene la limitación de la falta de generalización de los resultados al no haber realizado un control absoluto de variables extrañas ni disponer de un grupo de control. Una vez que esta metodología didáctica se ha puesto en marcha en el Colegio, se podría continuar la investigación con un análisis comparativo del empleo de la WebQuest en diferentes contextos, con una metodología empírico-experimental con medidas pretest-postest y un mayor control de las variables que pudieran influir en el aprendizaje cooperativo (amistad, predisposición al trabajo en grupo, etc.).

A continuación, se muestra en primer lugar algunas de las conclusiones obtenidas en investigaciones recientes sobre la eficacia del aprendizaje cooperativo y la WebQuest. En segundo lugar, se describe la metodología y los resultados de nuestra investigación. Y por último, se resumen algunas de las principales conclusiones obtenidas de nuestro estudio. 


\section{Estado de la cuestión}

\section{Eficacia del Aprendizaje Cooperativo}

Como es bien sabido el Aprendizaje Cooperativo (AC) consiste en el uso didáctico de grupos reducidos en los que los alumnos trabajan juntos para maximizar su propio aprendizaje y el de los demás. Es decir, una metodología que sistematiza a través de una serie de recursos didácticos, la necesidad de que los integrantes de un grupo trabajen juntos cooperando entre sí para realizar un trabajo (Jonhson, Jonhson y Smith, 1981). Una verdadera situación cooperativa fomenta en los miembros del grupo la certeza de que tienen que trabajar juntos para realizar la tarea; todas las metodologías que existen persiguen este objetivo. Por citar las metodologías más conocidas: método del rompecabezas (Aronson, Blaney, Sikes, Stephan y Snapp, 1978), equipo-juego-torneo (DeVries y Edwards, 1974), aprender juntos (Johnson y Johnson, 1975), controversia constructiva (Johnson y Johnson, 1979), grupo de investigación (Sharan y Sharan, 1976, 1992), estructuras de aprendizaje cooperativo (Kagan, 1985) e instrucción compleja (Cohen, 1994).

Los estudios que analizan el efecto del AC en el aprendizaje de los alumnos han señalado beneficios tanto en variables de tipo cognitivo como afectivo (Jonhson, Jonhson y Stanne, 2000; Jonhson and cols, 1981; Jonhson y Jonhson, 1990; Jonhson, Jonhson y Smith, 1998; Lara, 2001). En la mayoría de estos estudios se compara la eficacia del AC con enseñanzas de corte competitivo e individualista. Resumidamente estos son los resultados. Respecto a las variables de tipo cognitivo: el AC fomenta una mayor capacidad para resolver problemas, facilita la retención, adquisición de conceptos, mayor productividad, fomenta razonamientos de mayor calidad, así como la transferencia de lo aprendido. Y respecto a las variables de tipo afectivo: el AC fomenta mejores relaciones interpersonales entre los alumnos, apoyo social de los miembros del grupo, atribución del éxito o fracaso al trabajo realizado en el grupo, mayor curiosidad y motivación continuada, mayor compromiso con el aprendizaje.

\section{Eficacia de la WebQuest}

Una WebQuest es una actividad orientada a la investigación en la que parte o todos los recursos empleados provienen de Internet (Dodge, 1995). WebQuest fué ideada en 1995 por Bernie Dodge y Tom March, dos profesores de San Diego State University, para que los estudiantes hicieran buen uso del tiempo, se centraran en utilizar información más que en buscarla, y desarrollaran su pensamiento en los niveles de análisis, síntesis y evaluación (Dodge, 
1995). Según March (2004, 42) "una verdadera WebQuest es una estructura andamiada que emplea recursos de la red y una tarea auténtica para motivar la investigación de los estudiantes y el trabajo en grupo en la transformación de la información obtenida en un producto más sofisticado". En resumen los autores señalan a la WebQuest como un medio para promover el aprendizaje cooperativo y el andamiaje cognitivo. "El andamiaje es una estructura temporal empleada para ayudar a los estudiantes a ser más hábiles de lo que son. Una buena WebQuest construye andamiajes en el proceso del trabajo que deben seguir los alumnos para conseguir su objetivo" (Dodge, 2001, 58). El andamiaje cognitivo esta basado en los modelos de aprendizaje de experto (Bransford, Brown y Cocking, 2001).

A pesar de la rápida introducción de esta metodología en las aulas de todos los niveles educativos, existen pocos estudios que analicen su eficacia. Algunas investigaciones cuantitativas (Blanco, Fuente y Dimitriadis, 2004; MacGregor and Lou, 2004; Gaskill, McNulty y Brooks, 2006) y cualitativas (Hung, 2004; Angeli y Valanides, 2004; Almeida, Vesu y Ponte, 2003, 2004, 2005) llevadas a cabo sobre el trabajo de los alumnos en entornos didácticos con WebQuests, siguen avalando los mismos resultados que otros estudios desarrollados en contextos de trabajo colaborativo, en lo que se refiere a: mayor implicación de los alumnos al trabajar temas reales que son de su interés; predisposición al trabajo en grupo; preferencia de la ayuda entre iguales que la llevada a cabo por el profesor; motivación de los estudiantes en estos contextos; y eficacia del aprendizaje, alcanzando un alto desarrollo de estrategias cognitivas.

La investigación muestra que el andamiaje afecta positivamente en el resultado de los estudiantes al ofrecerles una estructura temporal que facilita una ejecución más allá de sus capacidades (Beriter y Scardamalia, 1984; Cho y Jonassen, 2002; Torrano y González-Torres, 2004; Martínez-Vicente y de la Fuente, 2004). No obstante hay pocos estudios que ofrezcan evidencia empírica de su efecto en el caso de la WebQuest. Mac-Gregor y Lou (2004) revelan que los andamios, en la forma de guías de estudio y mapas conceptuales, ayudan a los estudiantes que emplean una WebQuest. También, Lee, Pung y Lee (2005) señalan que los mapas conceptuales son una buena herramienta para organizar el trabajo de los estudiantes con una WebQuest. Por otro lado Angeli y Valanides (2004) analizan el efecto de los andamios ofre- 
cidos por Filamentality ${ }^{2}$. Este tipo de andamiaje sirve como una herramienta cognitiva que ayuda a los alumnos para organizar de un modo eficaz su pensamiento, y facilita recuperar información y usarla más tarde cuando la necesitan. Algunos estudios indican que la puesta en marcha de la WebQuest puede ser más difícil de lo que los diseñadores esperan. Una WebQuet debe diseñarse con cuidado y ofrecer suficientes actividades y materiales para que los estudiantes puedan emplearlos de modo eficaz (Lou y MacGregor, 2001; King, 2003).

\section{Metodología}

\section{Participantes}

Los participantes de este estudio son 24 alumnos varones de $4^{\circ}$ de Secundaria (16 años) del Colegio Erain (San Sebastián, España). Los alumnos tuvieron que realizar durante el mes de octubre de 2004 un trabajo para la asignatura de Geografía: un vídeo digital de investigación sobre diversos aspectos de la Geografía de la provincia de Guipúzcoa. Los alumnos se dividieron en grupos de 3 , en total se formaron 8 grupos.

\section{Contexto del estudio}

El trabajo que pedimos que realicen los alumnos es ciertamente complejo desde un punto de vista organizativo. En concreto, deben realizar un trabajo de investigación, un vídeo científico que resuma e integre lo estudiado e investigado, y además, deben trabajar de forma cooperativa.

Como decimos, el procedimiento para elaborar estos vídeos requiere un trabajo previo de investigación y planificación. Se pretende que los vídeos expresen el conocimiento adquirido en el trabajo de investigación realizado. La elaboración de cada vídeo debe cubrir las siguientes fases:

1) Fundamentación e investigación sobre el tema.

2) Guión del vídeo: información recogida en el trabajo de investigación y que será el texto de la narración del vídeo.

3) Storyboard o diseño de las tomas, planos y encuadres del vídeo. Se trata de aunar la información recogida en el trabajo de investigación y en la síntesis, de una forma visual.

\footnotetext{
${ }^{2}$ Filamentality (http://keithstanger.com/filamentality.html) facilita andamiaje electrónico a través de simples plantillas que ofrecen la posibilidad de organizar información online, y crear cinco tipos de actividades incluida la WebQuest.
} 
4) Revisión y reelaboración del trabajo hecho en los pasos previos. El profesor se encargará de revisar el material elaborado y proponer las sugerencias y modificaciones oportunas.

5) Filmación de las tomas una vez que han revisado el trabajo con el profesor.

6) Edición y montaje de las tomas y efectos visuales.

7) Y por último la grabación del audio, tanto la música como la voz de narración.

Cada grupo contó con un ordenador portátil Macintosh, una cámara de vídeo digital, el software iMovie para la edición del vídeo y un tiempo limitado para la realización de este trabajo. Los vídeos que realizaron pueden verse en la web:

http://www.videodigitaleducativo.com/

Para el éxito de nuestra propuesta se debe promover la autonomía del alumno dándole las pautas y guías necesarias para no perderse en el proceso. Por este motivo optamos por la WebQuest ya que permite dar las pautas y guías necesarias tanto para la elaboración de los vídeos como para el trabajo cooperativo.

Como es bien sabido son cinco los componentes esenciales del Aprendizaje Cooperativo (Johnson, Johnson y Holubec, 1994). En nuestra investigación hemos tratado de promoverlos fomentando:

1) la interdependencia positiva entre los miembros del grupo a través del resultado final que deben obtener al trabajar juntos, de los medios audiovisuales e informáticos empleados para la grabación y edición del vídeo, así como la distribución de roles o funciones. Para la producción y edición del vídeo se propone que los alumnos trabajen en grupos de tres personas. El trabajo de investigación se realiza de modo conjunto entre los miembros del grupo, y para el trabajo de elaboración del vídeo se reparte el trabajo según los roles de productor, narrador y cámara.

El productor es el que decidirá (aunque lo hagan entre los tres) cómo realizar el storyboard, dirigirá al cámara durante la filmación y decidirá en caso de duda cómo montar las imágenes.

El narrador será el que realizará la síntesis, grabará la voz, y hará las entrevistas que aparezcan en el vídeo.

El cámara filmará y manejará el ordenador durante la edición. 
2) La interacción estimuladora cara a cara a través del trabajo en grupo propuesto. El vídeo deben realizarlo conjuntamente los tres miembros del grupo.

3) Clara percepción de la responsabilidad individual para llegar a la consecución de los objetivos del trabajo en cada una de las fases propuestas.

4) Desarrollo de habilidades de trabajo en grupo a través de las instrucciones del trabajo propuestas en la WebQuest.

5) Evaluación del proceso grupal a través del calendario propuesto en la WebQuest para realizar cada una de las tareas. Los alumnos autoevalúan cómo van realizando el trabajo y controlan su actividad para ajustarse al tiempo establecido.

\section{Objetivos y contenidos}

En la Tabla 1 se muestran los objetivos que se persiguen con el trabajo que realizan los alumnos, así como los contenidos que se deben aprender.

Tabla 1.- Objetivos y contenidos del trabajo a realizar por los alumnos

\begin{tabular}{|c|c|c|}
\hline OBJETIVOS DIDÁCTICOS & Conceptuales & Actitudinales \\
\hline $\begin{array}{l}\text { - Desarrollar estrategias de búsqueda y selección de } \\
\text { información }\end{array}$ & $\begin{array}{l}\text { - Geografía de Guipúz- } \\
\text { coa }\end{array}$ & $\begin{array}{l}\text { - Valorar de los distintos modos de } \\
\text { vida en Guipúzcoa }\end{array}$ \\
\hline $\begin{array}{l}\text { - Producir un vídeo científico basado en una investi- } \\
\text { gación previa }\end{array}$ & $\begin{array}{l}\text { Proceso de } \\
\text { elaboración de un }\end{array}$ & $\begin{array}{l}\text { - Valorar la cooperación como forma } \\
\text { eficaz de trabajo en grupo }\end{array}$ \\
\hline $\begin{array}{l}\text { - Utilizar la aplicación iMovie para editar vídeos } \\
\text { - Fomentar el trabajo colaborativo entre los alumnos }\end{array}$ & $\begin{array}{l}\text { trabajo de investiga- } \\
\text { ción }\end{array}$ & $\begin{array}{l}\text { - Desarrollar la capacidad estética en } \\
\text { la edición del vídeo }\end{array}$ \\
\hline utilizando la WebQuest para definir y desarrollar la & \multicolumn{2}{|l|}{ Procedimentales } \\
\hline actividad. & \multicolumn{2}{|c|}{-Buscar y seleccionar información } \\
\hline $\begin{array}{l}\text { - Fomentar un conocimiento cercano y real de la } \\
\text { Geografía de Guipúzcoa }\end{array}$ & \multicolumn{2}{|c|}{$\begin{array}{l}\text { - Leer libros y recursos de Internet sobre el tema de la investiga- } \\
\text { ción }\end{array}$} \\
\hline - Aprender a realizar un trabajo de investigación & \multicolumn{2}{|c|}{ - Trabajar cooperativamente con los miembros del grupo } \\
\hline $\begin{array}{l}\text { - Trabajar cooperativamente a lo largo de todo el } \\
\text { proceso }\end{array}$ & \multicolumn{2}{|c|}{$\begin{array}{l}\text { - Utilizar una cámara de vídeo digital y software de edición de } \\
\text { vídeo }\end{array}$} \\
\hline
\end{tabular}




\section{La WebQuest}

Se decidió emplear la WebQuest para guiar el trabajo del alumno tanto en el proceso de indagación científica, como en la elaboración del vídeo, ofreciendo pautas para trabajar cooperativamente. La dirección de dicha WebQuest es:

http://www.erain.es/vde/produccion3.asp

Una WebQuest básicamente consiste en presentar al alumno una situación problemática que debe afrontar navegando por la red, buscando y seleccionando información, de forma guiada a partir de una serie de recursos propuestos por el profesor. Se les organiza en grupos, se les asignan roles y tienen que elaborar un producto que va desde una presentación o un documento, hasta una escenificación teatral o un guión radiofónico, un vídeo, etc.

El contenido de las WebQuest puede ser cualquiera. Su uso está teniendo una gran acogida en la escuela para el desarrollo del currículo oficial, así como para el desarrollo de temas transversales que favorecen unos modos de trabajar en el aula más colaborativos.

Esta metodología de trabajo en el aula persigue el desarrollo de competencias relacionadas con la indagación científica, como son: la capacidad de análisis, síntesis y evaluación de la información, de iniciativa y toma de decisiones, capacidad de observación y de ajuste a hechos, evidencias y datos concretos, de simplificar lo complejo sin perder la visión global, de desarrollo del pensamiento crítico, de planificación y organización, de ejecución y evaluación de lo planificado. Competencias todas ellas que ayudan a los alumnos a asimilar los conocimientos, integrarlos, relacionarlos con otros y así conseguir un aprendizaje más sólido. La WebQuest ha sido señalada como una estrategia que ayuda a desarrollar las habilidades del s. XXI por ser capaz de promover estas capacidades de indagación empleando la red (Lara, 2003). 


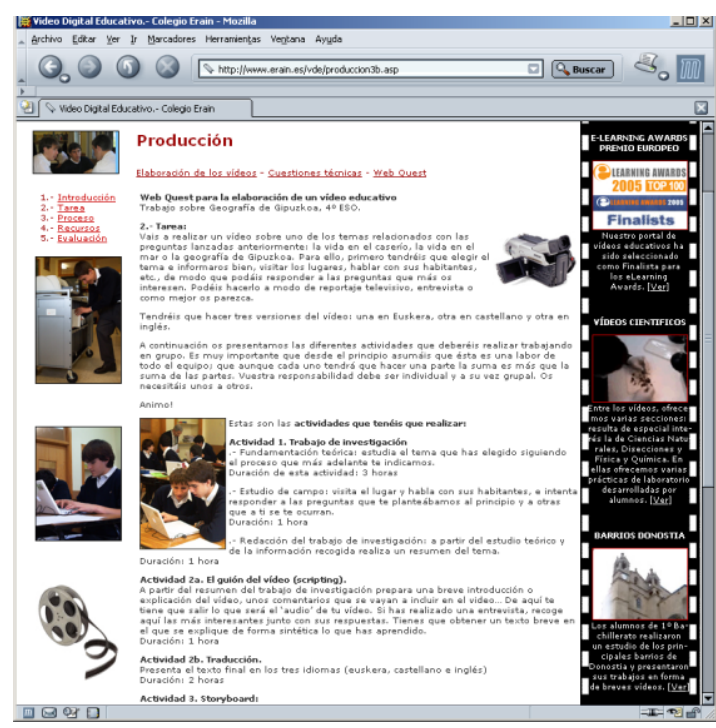

Figura 1.- Sección Tarea de la WebQuest

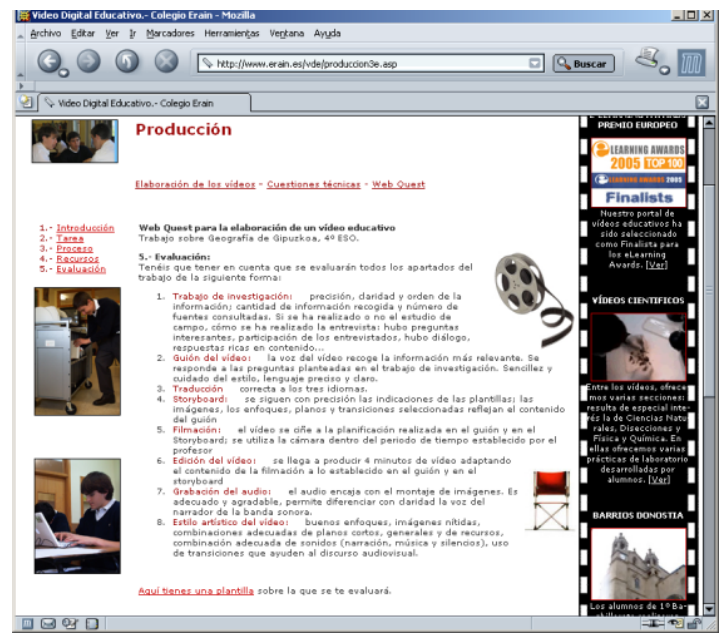

Figura 3.- Sección Evaluación de la WebQuest

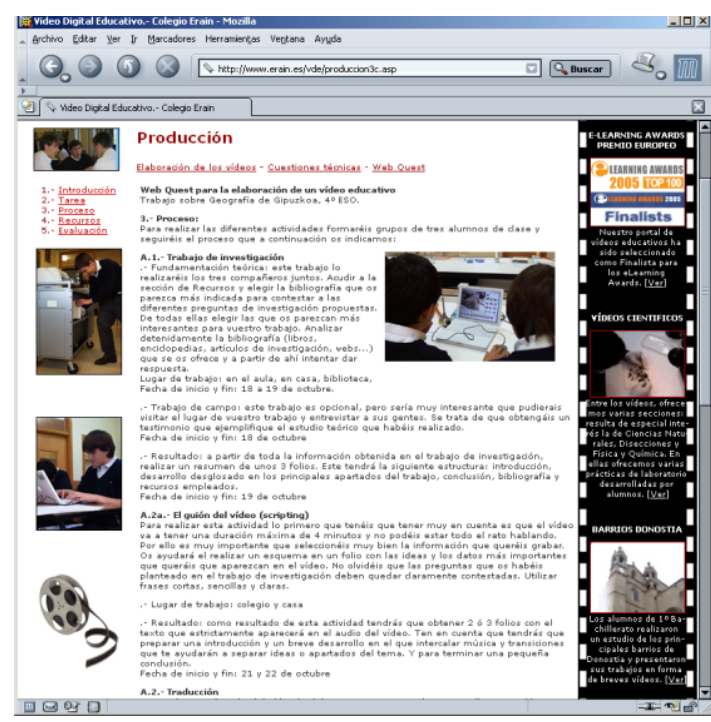

Figura 2.- Sección Proceso de la WebQuest

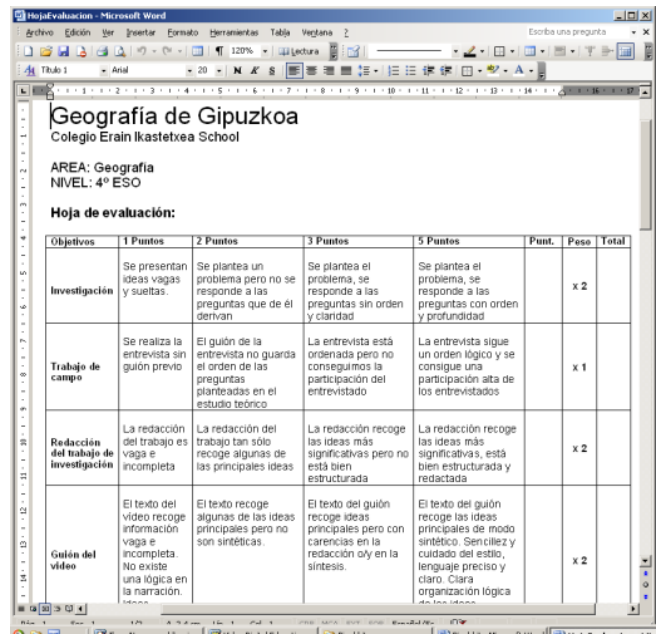

Figura 4.- Plantilla de evaluación

Queremos destacar dos rasgos importantes de la WebQuest que contribuyen al proceso de aprendizaje de los alumnos. Nos referimos tanto a la indagación científica y aprendizaje autónomo como al aprendizaje cooperativo (Repáraz, Lara, Mir y Orobiogoikoetxea, 2006, pp. 117-119):

- La indagación científica y el aprendizaje autónomo se facilita gracias a las guías y pautas (scafolding) ofrecidas a lo largo de toda la WebQuest para guiar el trabajo de los alumnos. En concreto y muy especialmente, gracias a la descripción detallada del trabajo que tienen que realizar, a los pasos que deben seguir y a las pautas de evaluación. Esto se describe respectivamente en los apartados de Tarea, Proceso y Evaluación de nuestra WebQuest (ver Figuras 1, 2 y 3). En el apartado “Tarea” se describe sucintamente en 
qué consiste el trabajo y las tareas que los alumnos deben realizar: trabajo de investigación, guión del vídeo, traducción del guión a las tres lenguas, storyboard, filmación, edición del vídeo y grabación del audio. En el apartado "Proceso" se describe en detalle qué tienen que hacer en cada tarea, el tiempo aproximado para su realización así como la fecha en la que debe estar realizado. En el apartado "Evaluación" de la WebQuest se presenta una plantilla (rubric) en la que se detallan cuáles van a ser los criterios de la evaluación final del trabajo (ver plantilla en Figura 4 y en la URL: http://www.erain.es/vde/recursos/HojaEvaluacion.doc)

- El aprendizaje cooperativo se intenta promover a través de la distribución y definición de los roles que los alumnos deben asumir. Los alumnos trabajan en grupos de tres personas, distribuyendo el trabajo en tres funciones distintas y necesarias entre sí. Esta distribución de tareas se hace especialmente necesaria en las fases de elaboración del vídeo.

\section{Pregunta de investigación}

La idea que guía el análisis de este artículo es que la WebQuest fomenta el necesario andamiaje para el trabajo cooperativo y autónomo de los estudiantes. La pregunta de investigación es: ¿La WebQuest fomenta el trabajo cooperativo y autónomo de los alumnos de secundaria (16 años) cuando realizan un vídeo científico?

\section{Recolección de datos y análisis}

El objetivo de introducir la WebQuest no es otro que el de ayudar y guiar a los alumnos tanto en su trabajo de investigación como en la elaboración de los vídeos para que trabajen de forma cooperativa. Por este motivo, los cuestionarios elaborados buscan analizar cómo han trabajado los grupos, si lo han hecho o no de forma cooperativa, distribuyendo las tareas y trabajos, y si han tenido las guías suficientes para realizar su trabajo de investigación y producción de los vídeos. Se han elaborado dos cuestionarios para recoger estos datos.

El primer cuestionario esta dividido en tres bloques de preguntas. Este cuestionario lo responde individualmente cada alumno, por tanto se recogen 24 cuestionarios.

* El primer bloque con once preguntas en una escala de seis puntos, pregunta a los alumnos si han trabajado de forma cooperativa. 
* El segundo bloque del cuestionario tiene cinco preguntas en una escala de cinco, y pide al alumno una valoración general de este modo de trabajar.

* El tercer bloque con cuatro preguntas abiertas, cuestiona a los alumnos sobre el formato del trabajo -realización del vídeo- frente al modo tradicional en lápiz y papel.

El segundo cuestionario, formado por preguntas de respuesta abierta, cuestiona a los alumnos sobre los aspectos que el grupo ha hecho bien y mal para trabajar juntos. Igualmente se pregunta por la calificación que esperan obtener en el trabajo. Este cuestionario lo contestan de mutuo acuerdo los tres integrantes del grupo, por tanto se recogen ocho cuestionarios, uno por cada grupo.

\section{Resultados}

\section{Primer cuestionario}

Primer bloque: Rasgos del trabajo cooperativo

Los resultados obtenidos en este primer bloque de preguntas reflejan que la mayoría de los alumnos han trabajado cooperativamente (ver Gráfico 1). Teniendo en cuenta que el cuestionario está en una escala de seis puntos, el 0 supone una frecuencia nula y el 5 una frecuencia permanente, la media obtenida en estas once primeras preguntas ha sido 3,85 (ver Tabla 2). Por otro lado el Alfa de Cronbach es 0.78. Todos estos datos permiten observar la consistencia de las respuestas dadas por los alumnos, así como la tendencia a una valoración positiva de las conductas cooperativas. En términos generales puede afirmarse que los alumnos perciben que han trabajado de forma cooperativa. 


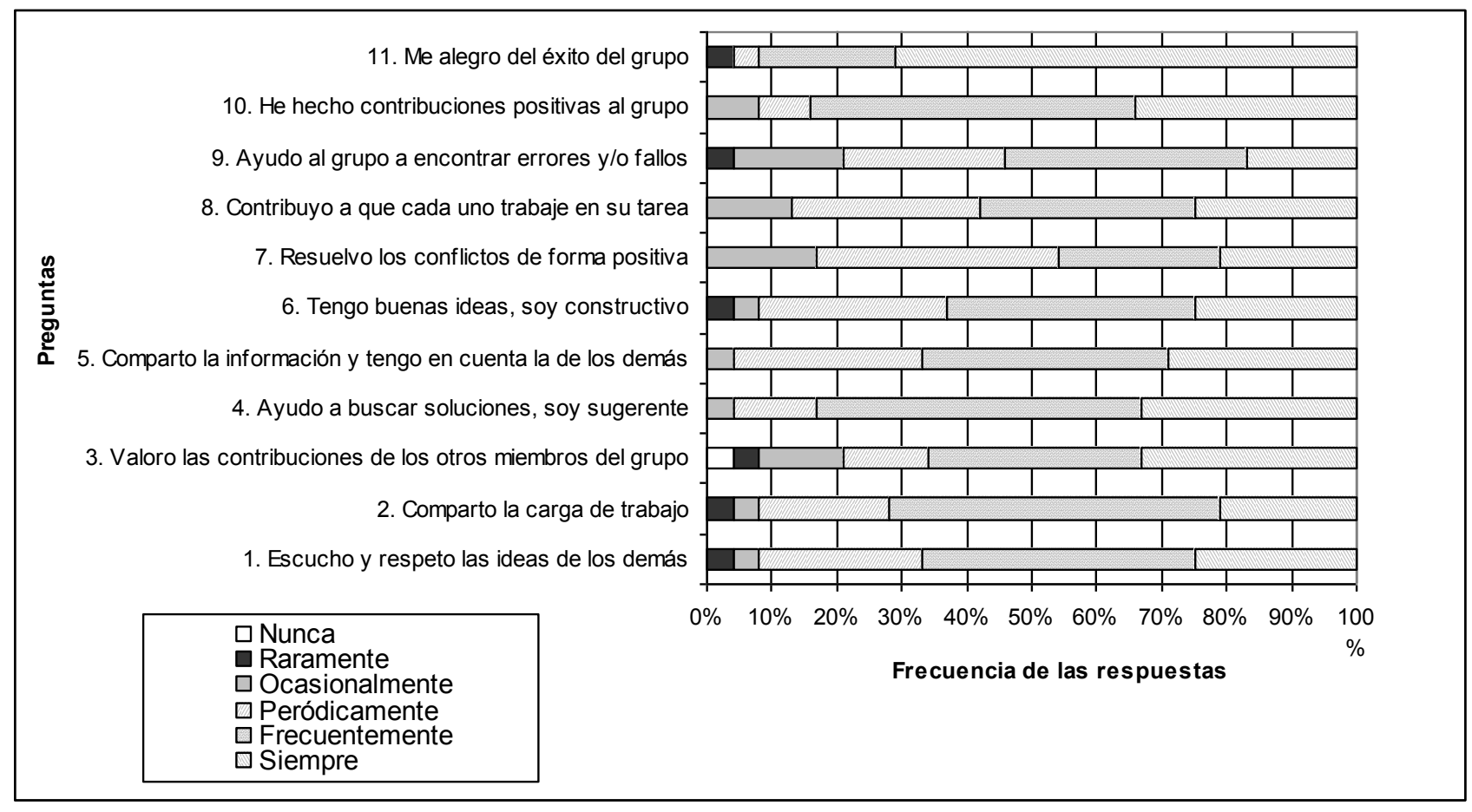

Gráfico 1.- Rasgos del trabajo cooperativo

Analizando de un modo detallado las respuestas, podemos observar que los ítems mejor valorados han sido (ver Tabla 2):

* ítem 4 "Ayudo a buscar soluciones, soy sugerente" $(\bar{X}=4.13, \mathrm{SD}=0.79)$

* ítem $5(\bar{X}=3.92, \mathrm{SD}=0.88)$ "Comparto la información y tengo en cuenta la de los demás"

* ítem 10 "He hecho contribuciones positivas al grupo" $(\bar{X}=4.08, \mathrm{SD}=0.88)$

* ítem11 "Me alegro del éxito del grupo" $(\bar{X}=4.55, \mathrm{SD}=0.93)$.

Todos estos ítems recogen conductas muy características del aprendizaje cooperativo como son la participación activa en el desarrollo del trabajo grupal, la ayuda a los demás miembros del grupo y el disfrute con los éxitos del grupo. 
Tabla 2.- Estadísticos descriptivos de la primera encuesta (I parte).

\begin{tabular}{lccccc}
\hline \multicolumn{1}{c}{ Preguntas/respuestas } & n & Mean & SD & Minimun & Maximun \\
\hline 1. Escucho y respeto las ideas de los demás & 24 & 3,79 & 1.02 & 1 & 5 \\
\hline 2. Comparto la carga de trabajo & 24 & 3,75 & 0.94 & 1 & 5 \\
\hline $\begin{array}{l}\text { 3. Valoro las contribuciones de los otros miembros } \\
\text { del grupo }\end{array}$ & 24 & 3,71 & 1.42 & 0 & 5 \\
\hline 4. Ayudo a buscar soluciones, soy sugerente & 24 & 4,13 & 0.79 & 2 & 5 \\
\hline 5. Comparto la información y tengo en cuenta la de & 24 & 3,92 & 0.88 & 2 & 5 \\
\hline$\quad$ los demás & & & & & 1 \\
\hline 6. Tengo buenas ideas, soy constructivo & 24 & 3,75 & 1.03 & 5 \\
\hline 7. Resuelvo los conflictos de forma positiva & 24 & 3,50 & 1.02 & 2 & 5 \\
\hline 8. Contribuyo a que cada uno trabaje en su tarea & 24 & 3,71 & 0.99 & 2 & 5 \\
\hline 9. Ayudo al grupo a encontrar errores y/o fallos & 24 & 3,46 & 1.01 & 1 & 5 \\
\hline 10. He hecho contribuciones positivas al grupo & 24 & 4,08 & 0.88 & 2 & 5 \\
\hline 11. Me alegro del éxito del grupo & 24 & 4,54 & 0.93 & 1 & 5 \\
\hline
\end{tabular}

Escala de seis puntos: $0=$ nunca $1=$ raramente $2=$ ocasionalmente $3=$ periódicamente $4=$ frecuentemente $5=$ siempre

Igualmente, si nos detenemos a analizar los coeficientes de correlación de Spearman de los ítems (ver Tabla 3), podemos observar como existen correlaciones significativas $(\mathrm{p}<0.001)$ entre varios de las preguntas de esta parte del cuestionario. Por destacar lo más significativo, el ítem 10 "He hecho contribuciones positivas al grupo" correlaciona con los ítems 2 "comparto la carga de trabajo", 3 "Valoro las contibuciones de los otros miembros del grupo", 6 "Tengo buenas ideas, soy constructivo" y 9 "Ayudo al grupo a encontrar errores y/o fallos". El ítem 5 "Comparto la información y tengo en cuenta la de los demás" correlaciona con el ítem 1 "Escucho y respeto las ideas de los demás", e ítem 3 "Valoro las contibuciones de los otros miembros del grupo". Todos estos datos no hacen más que confirmar lo que afirmabamos anteriormente, la consistencia en las valoraciones positivas de los alumnos sobre su percepción de haber trabajado de forma cooperativa. 
Tabla 3.- Coeficientes de correlación de Spearman (I parte).

\begin{tabular}{|c|c|c|c|c|c|c|c|c|c|c|c|}
\hline Preguntas/respuestas & 1 & 2 & 3 & 4 & 5 & 6 & 7 & 8 & 9 & 10 & 11 \\
\hline 1. Escucho y respeto las ideas ... & - & 0.21 & $0.54^{* *}$ & 0.15 & $0.65^{* *}$ & 0.22 & $0.47^{*}$ & 0.33 & 0.14 & 0.22 & 0.22 \\
\hline 2. Comparto la carga de trabajo & & - & $0.48^{*}$ & 0.10 & 0.36 & $0.44^{*}$ & 0.11 & 0.09 & 0.25 & $0.44^{* *}$ & $0.52^{* *}$ \\
\hline 3. Valoro las contribuciones ... & & & - & -0.11 & $0.54^{* *}$ & $0.48^{*}$ & 0.18 & -0.20 & 0.19 & $0.43^{* *}$ & 0.29 \\
\hline 4. Ayudo a buscar soluciones ... & & & & - & -0.04 & 0.33 & 0.34 & $0.51^{* *}$ & 0.24 & 0.40 & $0.47^{* *}$ \\
\hline 5. Comparto la información ... & & & & & - & 0.19 & 0.23 & 0.35 & 0.24 & 0.24 & 0.14 \\
\hline 6. Tengo buenas ideas ... & & & & & & - & 0.29 & 0.09 & 0.17 & $0.66^{* *}$ & 0.31 \\
\hline 7. Resuelvo los conflictos ... & & & & & & & - & 0.35 & 0.00 & 0.15 & 0.24 \\
\hline 8. Contribuyo a que cada ... & & & & & & & & - & 0.32 & 0.37 & 0.15 \\
\hline 9. Ayudo al grupo a ... & & & & & & & & & - & $0.48^{* *}$ & 0.11 \\
\hline 10. He hecho contribuciones ... & & & & & & & & & & - & 0.15 \\
\hline 11. Me alegro del éxito del grupo & & & & & & & & & & & - \\
\hline
\end{tabular}

${ }^{*} \mathrm{p}<0.05^{* *} \mathrm{p}<0.01$

\section{Segundo bloque: Valoración general}

Los resultados obtenidos en este segundo bloque de preguntas reflejan que los alumnos han valorado positivamente este modo de trabajar (ver Gráfico 2). Teniendo en cuenta que el cuestionario está en una escala de cinco puntos, donde el 1 supone una valoración insatisfactoria y el 5 una valoración excelente, la media obtenida en estas cinco preguntas ha sido de 3,93 (ver Tabla 4). Por tanto, en términos generales puede decirse que los alumnos hacen una valoración muy buena de esta forma de aprender.

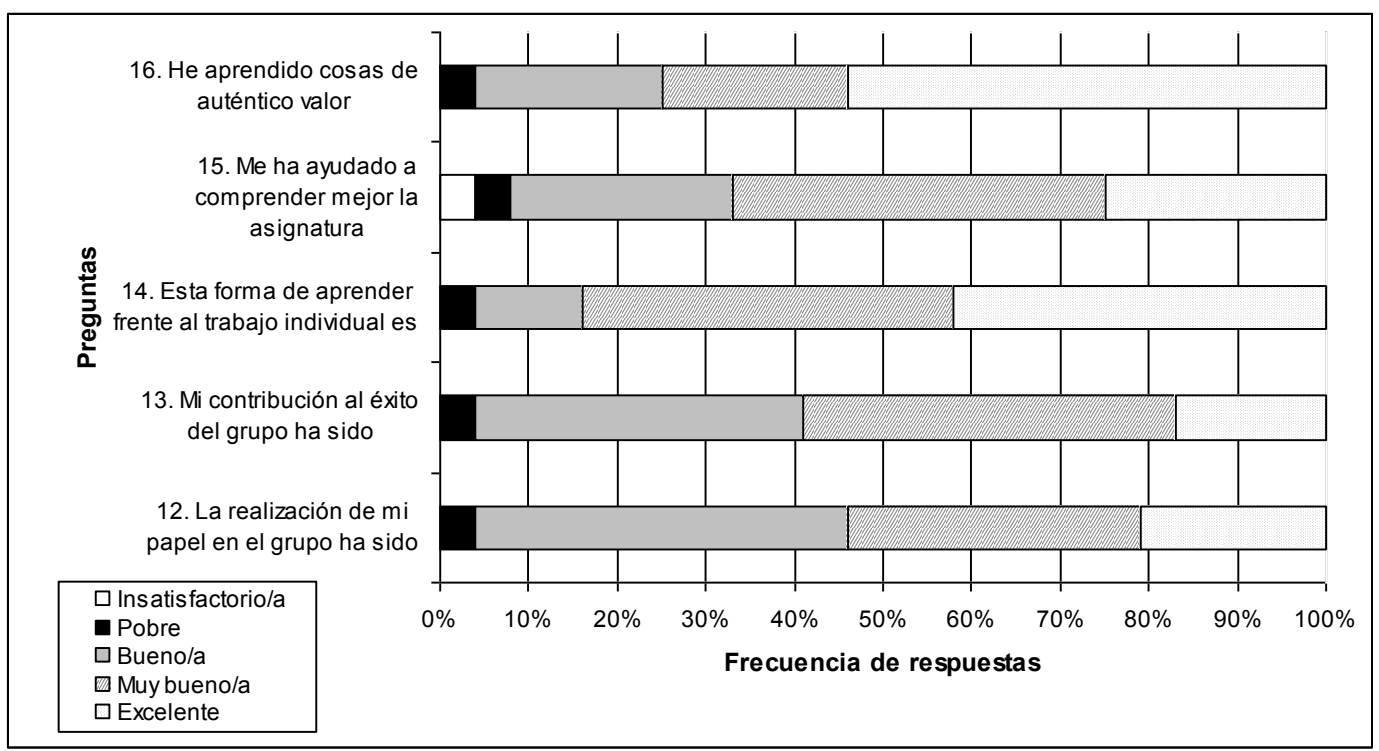

Gráfico 2 Valoración general 
Tabla 4.- Estadísticos descriptivos de la primera encuesta (II parte).

\begin{tabular}{lccccc}
\hline \multicolumn{1}{c}{ Preguntas/respuestas } & n & Mean & SD & Minimun & Maximun \\
\hline 12. La realización de mi papel en el grupo ha sido & 24 & 3.79 & 0.88 & 2 & 5 \\
\hline 13. Mi contribución al éxito del grupo ha sido & 24 & 3.67 & 0.82 & 2 & 5 \\
\hline 14. Esta forma de aprender frente al trabajo individual es & 24 & 4.21 & 0.83 & 2 & 5 \\
\hline 15. Me ha ayudado a comprender mejor la asignatura & 24 & 3.79 & 1.02 & 1 & 5 \\
\hline 16. He aprendido cosas de auténtico valor & 24 & 4.25 & 0.94 & 2 & 5 \\
\hline
\end{tabular}

Escala de cinco puntos: $1=$ insatisfactorio/a $2=$ pobre $3=$ bueno/a $4=$ muy bueno/a $5=$ excelente

Los ítems más valorados son el 14 "Esta forma de aprender frente al trabajo individual es" $(\bar{X}=4.21, \mathrm{SD}=0.83)$ y 16 "He aprendido cosas de auténtico valor" $(\bar{X}=4.25)$ que hacen referencia directamente al aprendizaje de los alumnos. En concreto, los alumnos hacen una valoración muy positiva del trabajo en grupo frente a otras formas de trabajo individual, esta metodología les ha ayudado a comprender mejor la asignatura, y al mismo tiempo, señalan que han aprendido cosas de auténtico valor. Las respuestas muestran que los alumnos han estado implicados en su proceso de aprendizaje y que además les ha gustado aprender de este modo.

\section{Tercer bloque: el trabajo con el vídeo digital}

En las Tablas 5, 6 y 7 se muestran las respuestas dadas por los 24 alumnos.

Respecto a la primera pregunta " ¿Te ha gustado presentar tu trabajo en formato vídeo?" todos los alumnos responden que sí (ver Tabla 5). La principal razón que dan es que se han divertido y disfrutado realizando el trabajo $(62,5 \%)$, además señalan que el producto final pueden enseñarlo a otras personas a través de la web del Proyecto $(16,6 \%)$. Otras respuestas minoritarias hacen referencia al hecho de aprender a manejar los ordenadores y las redes, aprender de una forma distinta, tener experiencias nuevas y una forma de aprender con más facilidad los contenidos. 
Tabla 5.- ¿Te ha gustado presentar tu trabajo en formato vídeo? ¿Por qué?

$\begin{array}{lcc}\text { Respuesta } & \text { Frecuencia } & \text { Porcentaje } \\ \text { Forma amena de trabajar } & 15 & 62,5 \% \\ \text { Producto final del vídeo } & 4 & 16,6 \% \\ \text { Aprender a manejar el ordenador, TIC } & 3 & 12,5 \% \\ \text { Otra forma de exponer los conocimientos y habilidades } & 2 & 8,3 \% \\ \text { Tener experiencias nuevas } & 2 & 8,3 \% \\ \text { Las ideas quedan más claras y ayuda a entenderlo mejor } & 1 & 4,1 \% \\ \text { Porque dejas volar la imaginación } & 1 & 4,1 \% \\ \text { La presentación del trabajo en clase es más sencilla que } & 1 & 4,1 \% \\ \text { con un trabajo escrito } & & \end{array}$

Las respuestas de la tercera pregunta muestran que sólo a un grupo le ha resultado difícil realizar el vídeo, más de la mitad de los grupos responde que no ha sido difícil (ver Tabla 6). Diez grupos señalan que algunas partes del vídeo han tenido cierta dificultad, la mayoría señala que la introducción del audio del vídeo ha sido complicada, así como el montaje de las imágenes o incluso la falta de tiempo para concluir mejor el trabajo.

Los grupos que han tenido dificultades han acudido a sus compañeros para resolver las dudas ( 7 respuestas), al profesor ( 5 respuestas), con paciencia y más trabajo y esfuerzo ( 2 respuestas) o no han resuelto la dificultad (1 respuesta). Estas respuestas muestran una vez más que han trabajado cooperativamente, ya que acuden con más frecuencia a los compañeros y al trabajo intenso, que al profesor para resolver las dificultades.

Tabla 6.- ¿Te ha resultado difícil realizar el vídeo? ¿cómo has resuelto las dificultades?

$\begin{array}{lcc}\text { Respuesta } & \text { Frecuencia } & \text { Porcentaje } \\ \text { No } & 13 & 54 \% \\ \mathrm{Si} & 1 & 4 \% \\ \text { Un poco, algunas partes del vídeo, falta de tiempo } & 10 & 42 \%\end{array}$

Todos los alumnos prefieren elaborar el trabajo en formato vídeo frente al formato de lápiz y papel, excepto un alumno que no le ha gustado el vídeo por dar mucho más trabajo. La respuesta mayoritaria de los alumnos, para preferir el trabajo en formato vídeo, ha sido que es una forma de aprender más divertida y entretenida, aunque les suponga más trabajo (75\%), así 
como aprender el contenido más fácilmente así como a retener mejor la información (33\%) (ver más respuestas en la Tabla 7).

Tabla 7.- ¿Es lo mismo hacer un trabajo en vídeo que en papel? ¿Tú qué prefieres? ¿Por qué?

$\begin{array}{lcc}\text { Respuesta } & \text { Frecuencia } & \text { Porcentaje } \\ \text { Es más divertido, interesante, menos aburrido } & 18 & 75 \% \\ \text { Facilidad de aprender y retener el contenido } & 8 & 33 \% \\ \text { Aprender a usar TIC } & 2 & 8,3 \% \\ \text { La presentación del contenido es más clara en vídeo } & 1 & 4,1 \% \\ \text { Video, en papel hemos hecho bastantes } & 1 & 4,1 \% \\ \text { Facilidad en la exposición del trabajo en clase } & 1 & 4,1 \%\end{array}$

La última pregunta de este bloque cuestiona a los alumnos sobre la facilidad de manejo de los portátiles Macintosh y la preferencia por los Macintosh o PC. La totalidad de los alumnos dicen que el manejo de los ordenadores así como el software para editar los vídeos ha sido fácil. El 63\% (15) de los alumnos prefieren los Macintosh y el 37\% prefieren los PC (9).

\section{Segundo cuestionario}

\section{Cosas que el grupo ha hecho bien para trabajar juntos}

La mayoría de los alumnos consideran que han distribuido bien las cargas del trabajo entre los miembros del grupo (87\%). Más de la mitad de los grupos considera que se ha puesto de acuerdo al tomar decisiones, contando con las opiniones de todos los miembros del grupo, escuchando y respetando las ideas de otros (62\%). Ambas conductas son fundamentales y caracterisiticas del aprendizaje cooperativo (ver Tabla 8). Uno de los objetivos que perseguimos con el empleo de la WebQuest era precisamente favorecer tanto un modo de trabajo cooperativo como pautar guías y pasos que ayudaran a los alumnos a trabajar con cierta autonomía. Las respuestas de los alumnos nos hace afirmar que se ha conseguido en la mayoría de los grupos. 
Tabla 8.- Valoración de conductas que han facilitado el trabajo en grupo

\begin{tabular}{lcc}
\multicolumn{1}{c}{ Opinión } & Frecuencia & Porcentaje \\
Distribución del trabajo, organización, trabajo en equipo & 7 & $87,5 \%$ \\
Ponerse de acuerdo. Respetar las ideas de los demás & 5 & $62,5 \%$ \\
Disfrutar mientras trabajan juntos & 2 & $25 \%$ \\
Distribución del tiempo & 2 & $25 \%$ \\
Distribución del material & 1 & $12,5 \%$ \\
Hacer fácil el trabajo & 1 & $12,5 \%$ \\
Trabajar con velocidad & 1 & $12,5 \%$ \\
Trabajar con intensidad & 1 & $12,5 \%$ \\
Tener ideas claras del trabajo a realizar & 1 & $12,5 \%$
\end{tabular}

\section{Cosas que el grupo ha hecho mal para trabajar juntos}

$\mathrm{Al}$ menos cuatro grupos (50\%) consideran que ha habido una falta de previsión en el tiempo. Otras respuestas minoritarias hacen referencia a la dificultad para reunirse a trabajar, perder tiempo en discusiones, falta de esfuerzo, y dificultad para compartir el ordenador (ver Tabla 9).

Tabla 9.- Valoración de conductas que han dificultado el trabajo en grupo

$\begin{array}{lcc}\text { Opinión } & \text { Frecuencia } & \text { Porcentaje } \\ \text { Repartir mal el tiempo } & 4 & 50 \% \\ \text { Dificultad en los horarios para reunirse a trabajar } & 1 & 12,5 \% \\ \text { Perder tiempo en discusiones } & 1 & 12,5 \% \\ \text { Poco esfuerzo e intensidad en el trabajo } & 1 & 12,5 \% \\ \text { No compartir el ordenador } & 1 & 12,5 \%\end{array}$

\section{Expectativa de calificación}

Existe una alta correlación entre las notas que obtuvieron finalmente los alumnos por la realización de este trabajo y las notas que ellos esperaban obtener: Rho de Spearman=0,82. Aunque la $n$ es pequeña -sólo se ha calculado la correlación entre ocho grupos- se puede valorar este dato como una tendencia de que las notas obtenidas están en consonancia con las notas esperadas por los alumnos. Este hecho, podría atribuirse como una consecuencia de trabajar con la WebQuest. Nos referimos a que el trabajo bien pautado que marca la WebQuest ayuda al alumno a conocer bien lo que se espera de él, y a que éste pueda valorar mejor su trabajo. En este sentido, la WebQuest que diseñamos tiene un apartado que incluye los crite- 
rios de evaluación, detallando todos los aspectos que el profesor tendría en cuenta en la evaluación del trabajo final (ver Figura 3 y 4 ). Más información en: http://www.erain.es/vde/recursos/HojaEvaluacion.doc.

\section{Conclusiones}

Como indicábamos al inicio de este artículo, el Director del Colegio Erain estaba preocupado porque los alumnos estaban más interesados en producir el vídeo, que en hacer la investigación previa necesaria para el contenido del vídeo. Por ello buscaba la forma de dar pautas que guiaran a los alumnos. Los resultados de este estudio muestran como la WebQuest -diseñada para fomentar el aprendizaje autónomo y el trabajo cooperativo en la elaboración de los vídeos- ha sido eficaz para conseguir nuestro objetivo. No obstante, este estudio tiene sus limitaciones. Los resultados no pueden ser transferidos más allá del propósito con el que se inició: ofrecer una solución a un problema que tenían en el Colegio Erain con la producción de vídeos de los alumnos. Por un lado, el efecto de la motivación de emplear el vídeo ha podido influir en las percepciones y respuestas de los estudiantes. El empleo del vídeo digital es en sí motivante para los estudiantes, ya que implica un modo completamente diferente de llevar a cabo un trabajo frente al tradicional realizado en papel. Este modo de trabajar, como reconocen los estudiantes en las encuestas, requiere más esfuerzo que un formato de lápiz y papel pero les motiva más el empleo de las TIC, así como poder mostrar a sus compañeros de clase y familiares, a través de la web del colegio, el video realizado. Por otro lado, un número de variables que podrían haber afectado al aprendizaje cooperativo -como la predisposición a trabajar juntos- no han sido controladas a priori.

A pesar de las limitaciones indicadas respecto a la transferencia de los resultados, el Director del Colegio Erain y el profesor involucrados en esta investigación consideran haber conseguido su objetivo: encontrar en la WebQuest una metodología didáctica para mejorar el aprendizaje autónomo y el aprendizaje cooperativo de sus alumnos de Secundaria (16 años) en la producción de vídeos científicos. Además, muchos de los resultados son acordes con los señalados en la literatura sobre la eficacia del aprendizaje cooperativo:

- En concreto nos referimos tanto a variables cognitivas, como afectivas. Por un lado, respecto a variables cognitivas los datos muestran que los alumnos creen que aprenden 
y retienen con más facilidad los contenidos trabajados de esta forma. Existe una mayor implicación en la tarea, se esfuerzan más y buscan formas alternativas de encontrar información. Dicen aprender cosas de auténtico valor, que se facilita la comprensión de la asignatura, y que saben organizar el trabajo. Por otro lado, respecto a variables afectivas, los alumnos se muestran más motivados a trabajar en grupo y a elaborar un vídeo. Atribuyen el éxito al esfuerzo y trabajo realizado, ya que saben qué se espera de ellos y conocen los criterios con los que serán evaluados. Se fomentan mejores relaciones en el grupo, ya que la mayoría reconoce respetar las ideas de los demás, contribuir al éxito del grupo y acudir a sus compañeros cuando se presentan dificultades.

- Respecto a los resultados obtenidos sobre los beneficios del andamiaje, podemos afirmar que las pautas ofrecidas en la WebQuest para que los alumnos trabajen cooperativamente han sido suficientes. La mayoría de los estudiantes afirma haber sabido organizar y distribuir las tareas entre los miembros del grupo. No obstante, se ve necesario dar un mayor número de pautas para ayudarles a organizar el tiempo. Al parecer la información que se les ha dado (las fechas tope para entregar cada parte del trabajo, así como la indicación de los tiempos estimados para cada tarea) no ha sido suficiente. Para futuras versiones de la WebQuest sería conveniente introducir algún tipo de ayuda para guiar al alumno en este aspecto (Lou y MacGregor, 2001; King, 2003)..

Agradecimientos: Queremos agradecer a José Ignacio Mir, Director del Colegio Erain, su apoyo en la recogida de los datos. Y a Enrique Orobiogoikoetxea, profesor de Geografía en $4^{\circ}$ de ESO, haber seguido nuestras sugerencias en la aplicación de la WebQuest. Igualmente agradecemos los comentarios realizados por los dos revisores (peer review) ciegos de la revista. 


\section{Referencias}

Almeida, C., Vesu, F. y Ponte, J.P. (2003). WebQuest construction and implementation by mathematics student teacher: The case of a WebQuest to learn isometries. In A.M.Vilas, J.A.M.González y J.M. González (Coords.), Advances in TechnologyBased Education: Toward a Knowledge-Based Society (pp. 1396-1399) Junta de Extremadura: Badajoz.

Almeida, C., Vesu, F. y Ponte, J.P. (2004). Reflections of a student teacher on his construction and implementation of WebQuest to teach $7^{\text {th }}$ grade statistics. In R.Ferdin, (Eds.) Society for Information Technology and Teacher Education International Conference Annual: Proceedings of SITE 2004 (pp. 4353-4358). Norfolk, VA: AACE.

Almeida, C., Vesu, F. y Ponte, J.P. (2005). Factors of success in the implementation of mathematics WebQuests: Reflections of a student teacher. In Crawford (Ed) Society for Information Technology and Teacher Education International Conference Annual: Proceedings of SITE 2005 (pp. 3433-3437). Norfolk, VA: AACE.

Angeli, Ch. y Valanides, N. (2004). The effect of electronic scaffolding for technology integration on perceived task effort and confidence of primary student teachers. Journal of Research on Technology in Education. 37(1), 29-43.

Aronson, E., Blaney, N., Sikes, J., Stephan, C., y Snapp, M. (1978). The Jigsaw Classroom. Beverly Hills, CA: Sage.

Bereiter, C., y Scardaanalia, M. (1981). Teachability of reflective processes in written composition. Cognitive Science. 180(8), 173-190.

Blanco, S., de la Fuente, P y Dimitriadis, Y. (2004). Estudio de caso: uso de WebQuest en educación secundaria. [online] http://ryc.educaragon.org/files//webquest_secundaria.pdf(Septiembre, 2004).

Bransford, J. D.; Brown, A. L. y Cocking, R. R. (ed.) (2001). How People Learn. Brain, Mind, Experience, and School. Expanded Edition. Washington, D. C.: National Academy Press.

Cho, K., y Jonassen, D. (2002). The effects of argumentation scaffolds on argumentation and problem solving. Educational Technology Research anzd Developmnent, 50 (3), 5-22.

Cohen, E (1994). Designing Groupwork: Strategies for the Heretoregeneous Classrooms: Sociological Theory in Practice. New York: Teachers College Press. 
DeVries, D., y Edwards, K. (1974). Student Teams And Learning Games: Their Effects On Cross-Race And Cross-Sex Interaction. Journal of Educational Psychology, 66, 741749.

Dodge, B. (1995). Some thoughts about WebQuests. [online] http://edWeb.sdsu.edu/courses/edtec596/about_WebQuests.html (Agosto, 2003)

Dodge, B. (2001). FOCUS. Five Rules for Writing a Great WebQuest. Learning and Leading with Technology, 28 (8), 6-9, 58.

Gaskill, M., McNulty, A., y Brooks, D.W. (2006). Learning from WebQuests. Journal of Science Education and Technology, 15 (2), 133-136.

Hung, Ch. (2004). The use of WebQuest as a constructivist learning tool in secondary school geography in Singapore. Paper presented at National Educational Computing Conference 2004. [online] http://center.uoregon.edu/conferences/ISTE/NECC2004/about_NECC/default.php (Septiembre, 2004)

Johnson, D. W., y Johnson, R. (1975). Learning Together and Alone: Cooperative, Competitive, and Individualistic Learning. Boston: Allyn y Bacon. First edition, 1975.

Johnson, D. W., y Johnson, R. (1979). Conflict in the classroom: Controversy and learning. Review of Educational Research, 49, 51-70.

Johnson, D. W., Johnson, R. T. y Holubec, E. J. (1993). Cooperation in the classroom (6th ed.). Edina, MN: Interaction Book Company.

Johnson, D. W., Maruyama, G., Johnson, R., Nelson, O. y Skon, L. (1981). Effects of Cooperative, Competitive, And Individualistc Goal Structures On Achievement: A MetaAnalysis, Psychololical Bulletin, 89, 47-62.

Johnson, D.W., Johnson, R.T. y Smith, K. (1998). Controversy within Decision-Making Sitations. In M.Rahim (Ed). Managing Conflict: An Interdisciplinary Approach. New York: Praeger Publishing Co.

Jonhson, D.W. y Johnson, R.T. (1990). Using Cooperative Learning in Math. En N. Davidson (Ed.), Cooperative Learning in Mathematics: A Handbook for Teachers (pp. 219250) New York: Addison-Wesley.

Jonhson, D.W., Johnson, R. y Stanne, M. B. (2000, May). Cooperative Learning Methods: A Meta-Analysis, [online]. The Cooperative Learning Center at The University of Minnesota. Disponible: http://clcrc.com/pages/cl=methods.html [2001, Diciembre]

Jonhson, D.W., Johnson, R.T. y Smith, K. (1981). Active learning: cooperation in the classroom. Interaction Book Company. Edina: $\mathrm{MN}$. 
Kagan, S. (1985). Cooperative learning resources for teachers. Riverside, CA: University of California at Riverside.

King, K. P. (2003). The WebQuest as a means of enhancing computer efficacy. (ERIC Document Reproduction Service No. ED474439)

Lara, S. (2001). Una estrategia eficaz para fomentar la cooperación. Estudios sobre Educación, 1, 99-110.

Lara, S. (2003). WebQuest: The use of Internet to Introduce Inquiry-Based Learning, Cooperative Learning and 21th Century Skills. En Méndez Vilas, A. y Mesa González, J.A. (coord). Advances in Technology-Based Education: Toward a Knowledge-Based Society (pp 1178-1183). Vol. 2. Junta de Extremadura. Consejería de Educación, Ciencia y Tecnología.

Lee, F., Pun, S., Yang, H., y Lee, H. (2005). Effectiveness of Using Concept Mapping for Inquiry-based Project Design and Development. In C. Crawford et al. (Eds.), Proceedings of Society for Information Technology and Teacher Education International Conference 2005 (pp. 1717-1722). Chesapeake, VA: AACE

Lou, Y. y MacGregor, K. (2001). Leaning with Internet resources: Task structure and group classroom. Paper presented at the $12^{\text {th }}$ International Conferenci of Society for Information Technology and Techer Education, March, Orlando, FL.

MacGregor, S.K y Lou, Y. (2004). Web-Based Learning: How Task Scaffolding and WebSite Design Support Knowledge Acquisition. Journal of Research on Technology in Education. 37(2), 161-175.

March, T. (1995). What's on the Web? Computer-Using Educators' Newsletter July/ August [online] http://www.ozline.com/learning/webtypes.html [Agosto, 2003].

March, T. (2004). The learning power of WebQuest. Educational Leadership, 61 (4), $42-47$.

Martínez, J. M. y De la Fuente, J. (2004). Self-Regulation of learning trough the Pro-Regula Program. Electronic Journal of Research in Educational Psychology, 2(2), 145-156. www.investigacion-psicopedagogica.org/revista/english

Repáraz, Ch., Lara, S., Mir, J.I. y Orobiogoikoetxea, E. (2006) Empleo de WebQuest para la elaboración de vídeos científicos en geografía ( $4^{\circ}$ de la ESO). La indagación científica y el aprendizaje cooperativo. Iber. Didáctica de las Ciencias Sociales, Geografía e Historia.. 49, July, 109-122.

Sharan, S., y Sharan, Y. (1976). Small Group Teaching. Englewood Cliffs, NJ: Educational Technology Publications. 
Sharan, S., y Sharan, Y. (1992). Group Investigation: Expanding Cooperative Learning. New York: Teacher's College Press.

Torrano, F. y González-Torres, M. C. (2004). Self-regulated learning: Current and futures directions. Electronic Journal of Research in Educational Psychology, 2 (1), 1-34. www.investigacion-psicopedagogica.org/revista/english 